\title{
Dynamical Network of HIV-1 Protease Mutants Reveals the Mechanism of Drug Resistance and Unhindered Activity
}

Rajeswari Appadurai and Sanjib Senapati*

BJM School of Biosciences and Department of Biotechnology,

Indian Institution of Technology Madras, Chennai 600 036, India.

Tel: +91-44-22574122, Fax: +91-44-22574102, E-mail: sanjibs@iitm.ac.in

\section{Supporting information:}

Table S1: Kinetic parameters of drug and substrate binding to wild type and HIV-1 protease variants. Data adapted from Ref. 6 in main text.

\begin{tabular}{|c|c|c|c|c|}
\hline Ligand & WT & $\mathrm{AM}$ & NAM & ANAM \\
\hline \multicolumn{5}{|c|}{$\begin{array}{l}\text { Inhibition constant, } K_{i}(\mathrm{~nm}) \text { for two representative FDA-approved HIV drugs. Fold increase in } \\
\text { drug resistance of the variants with respect to the WT are given in parenthesis. }\end{array}$} \\
\hline Indinavir & $0.543(1)$ & $4.4(8)$ & $481(890)$ & $1102(2030)$ \\
\hline Ritonavir & $0.027(1)$ & $0.91(33)$ & $340(12600)$ & $2107(78000)$ \\
\hline \multicolumn{5}{|c|}{$\begin{array}{l}\text { Substrate catalytic efficiency, } K_{c a t} / K_{m}\left(\mathrm{~s}^{-1} \mu \mathrm{M}^{-1}\right) \text { of WT and HIV protease variants. Fold increase } \\
\text { in catalytic efficiency of the variants with respect to the WT are given in parenthesis. }\end{array}$} \\
\hline CA-p2 & $0.84(1)$ & $2.48(2.95)$ & $0.96(1.14)$ & $1.47(1.75)$ \\
\hline
\end{tabular}


a)

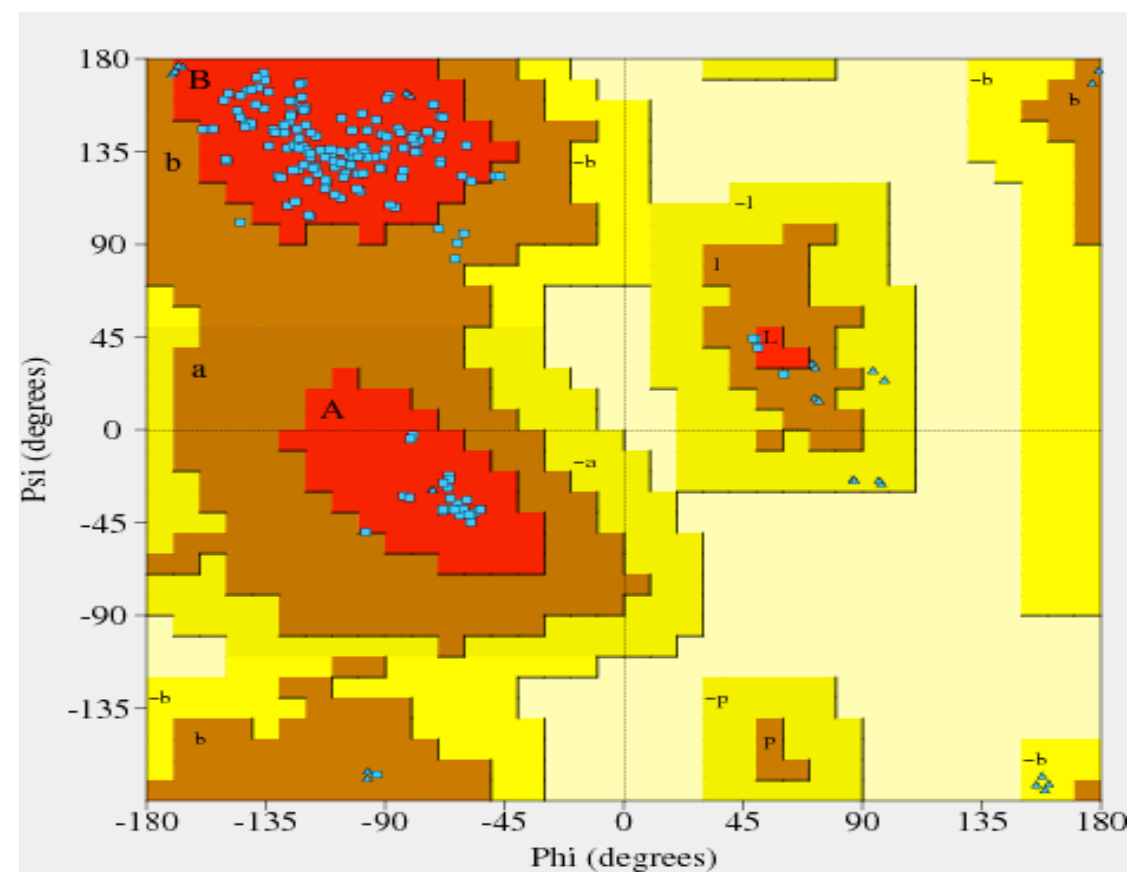

b)

\begin{tabular}{|l|l|l|}
\hline Stereochemical parameter & Score & $\begin{array}{l}\text { Average } \\
\text { score }\end{array}$ \\
\hline Dihedral angles:- & & \\
$\quad$ Phi-psi distribution & -0.27 & \\
Chil-chi2 distribution & -0.25 & -0.08 \\
Chil only & 0.18 & \\
Chi3 \& chi4 & 0.48 & \\
Omega & -0.02 & \\
\hline Main-chain covalent forces:- & & \\
$\quad$ Main-chain bond lengths & -0.21 & -0.26 \\
Main-chain bond angles & -0.31 & \\
\hline Overall average G-factor & & -0.17 \\
\hline
\end{tabular}

Figure S1: PROCHECK validation of the model structures of protease variants: a) Ramachandran plot of the ANAM variant. Blue dots indicate the Phi-Psi angle distribution of the protease residues. Red region represents the most favored region, brown = allowed region, yellow $=$ generously allowed region, light yellow $=$ disallowed region in the Ramachandran plot. b) Calculated stereochemical parameters and average G-factor of protease ANAM variant that provide a measure of how unusual a given stereochemical property is. Values below -0.5 represent unusual; values below -1.0 represent highly unusual distribution. 

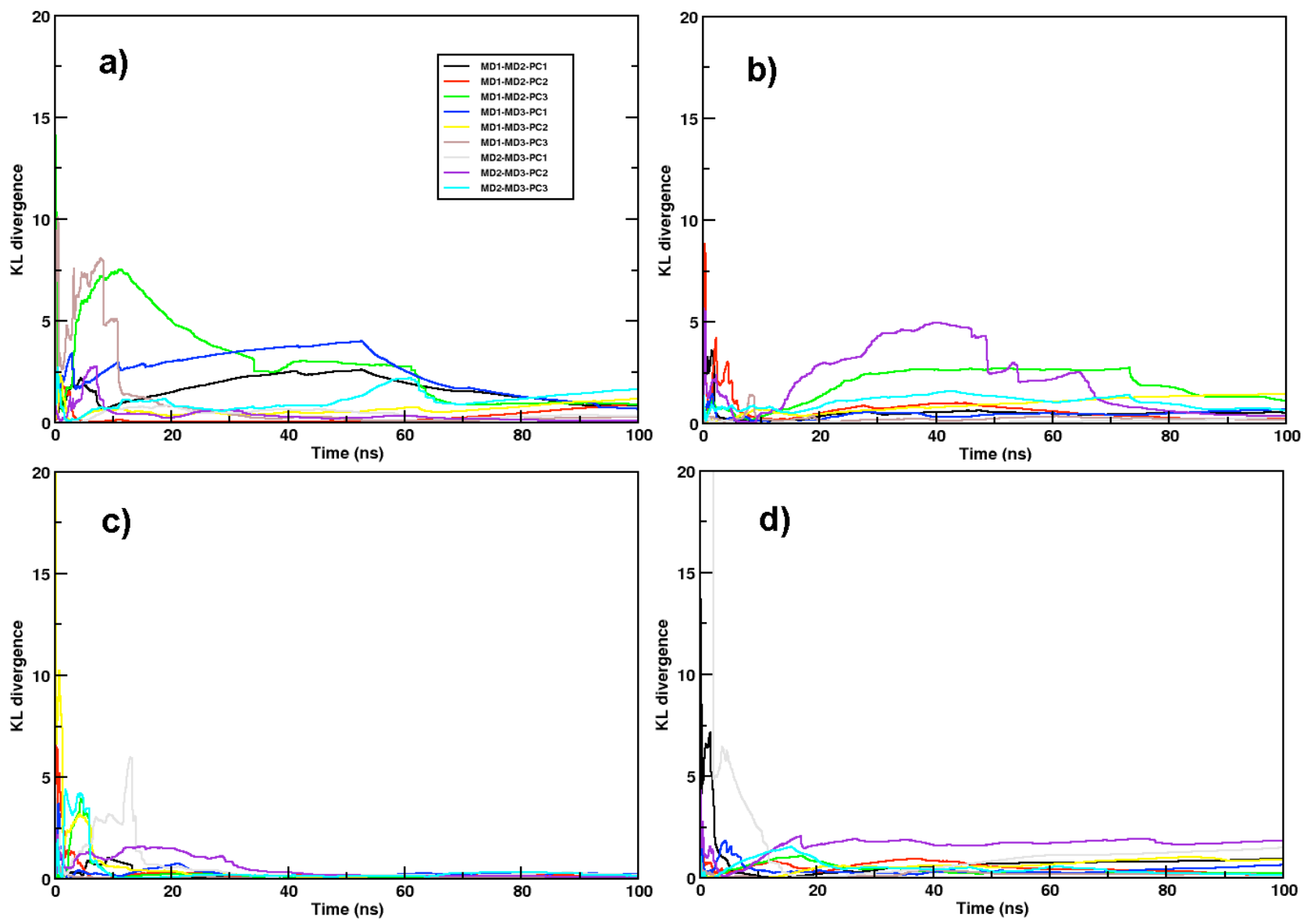

Figure S2: Convergence of MD simulation data. Kullback-Leibler (KL) divergences are shown for the principal components of protein motions from three independent simulations as a function of time. Divergence analysis is done for all possible pairs of MD simulations, i.e. MD1 and MD2, MD2 and MD3, MD1 and MD3, and first three principal components are considered. Results are shown for a) WT, b) AM, c) ANAM and d) NAM systems. 
a)

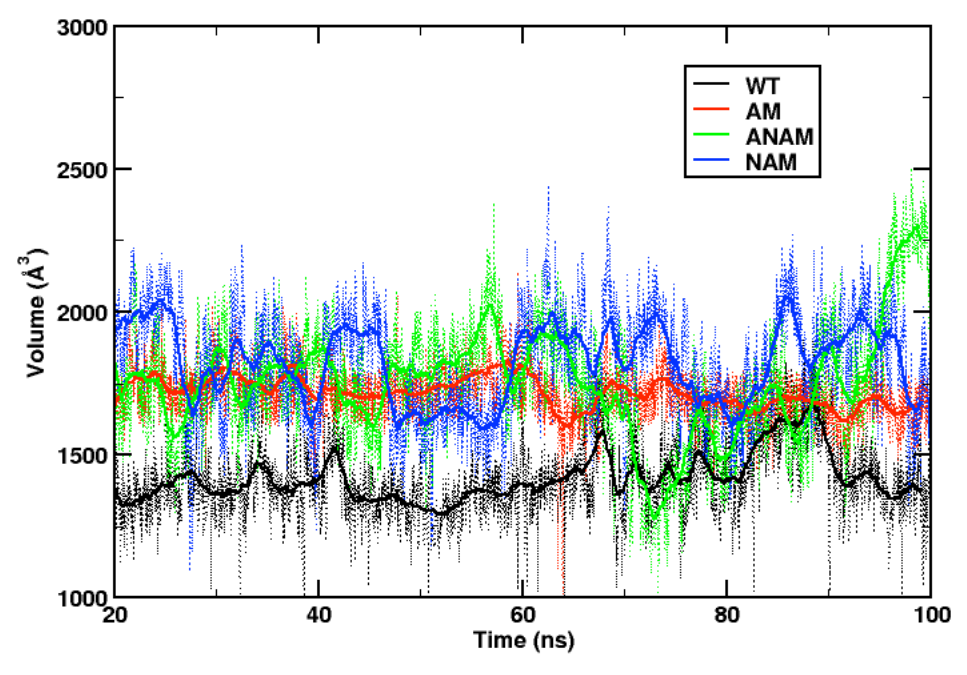

b)
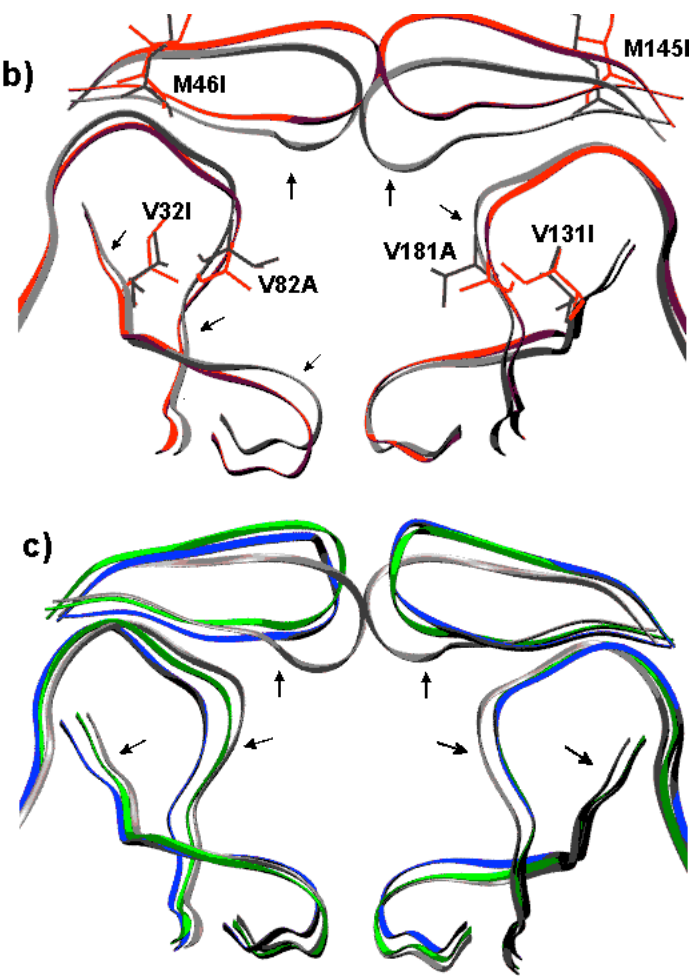

Figure S3: Comparison of active site pocket volume in WT and mutant proteases. a) Time dependence of pocket volume is shown for WT (black), AM (red), ANAM (green), and NAM (blue). While the dotted line represents the volume calculated for every 20ps frames of the trajectory, the solid line represents the running average along the simulation time scale. The mean volume of the active site pocket in WT, AM, ANAM and NAM were found to be 1417.73 $\pm 128,1716.71 \pm 93,1768.37 \pm 217$ and $1860.61 \pm 185 \AA^{3}$ by averaging over the three sets of MD simulation data. b) Superposition of time averaged structures of WT (gray) and AM (red) reveals that the walls lining the active site pocket in AM expanded due to mutations. For clarity only the surrounding wall region of the active site pocket is shown as ribbons. The mutated residues of the active site are shown as stick representation. c) Superposition of average structures of ANAM (green) and NAM (blue) onto WT structure (gray). In b and c, the arrows indicate the direction of shifting of walls lining the pocket from WT to mutant proteases. 


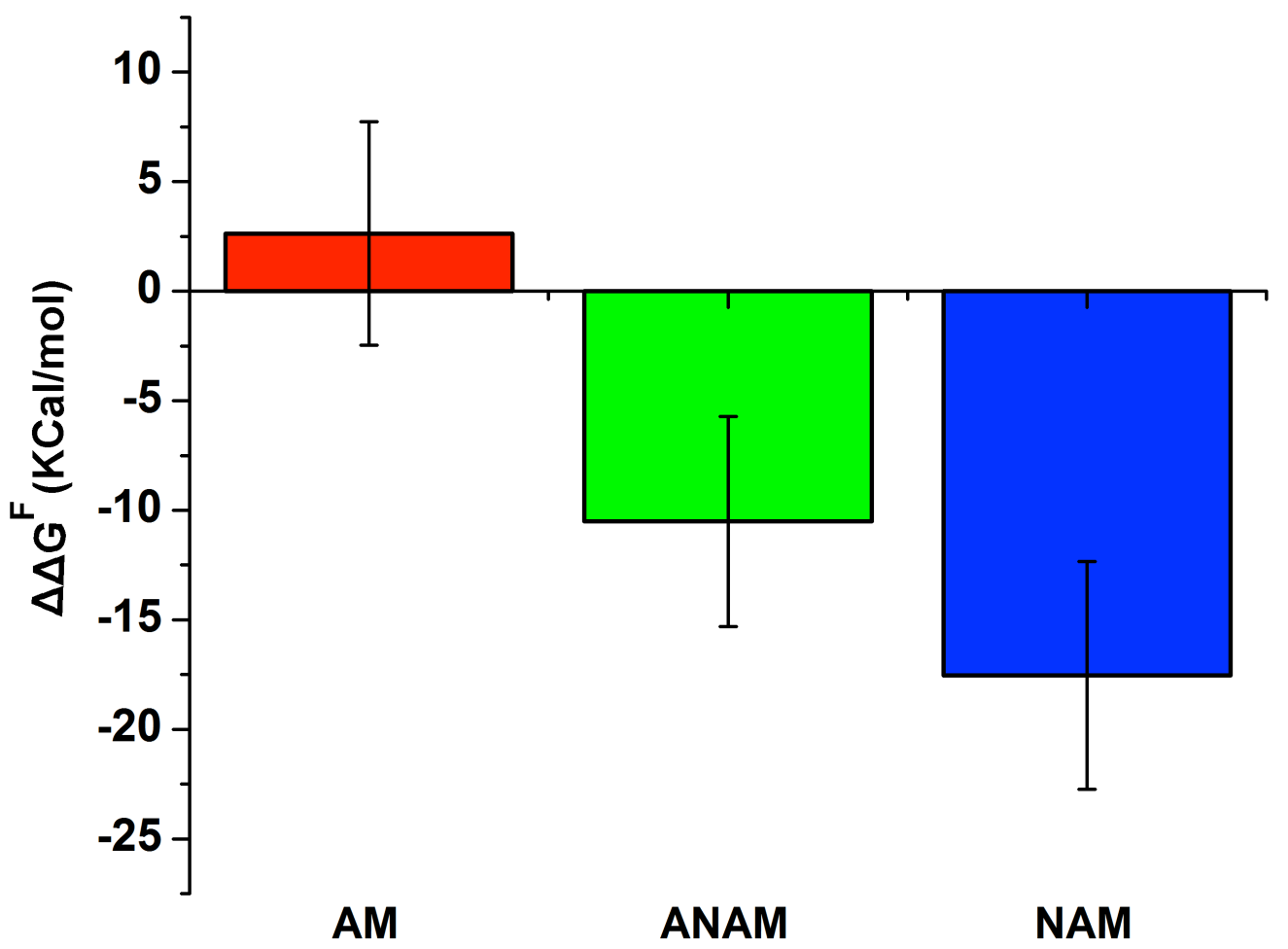

Figure S4: Relative stability of protease variants with respect to the WT, calculated as the change in free energy of folding of the variant minus WT. Hence, the positive $\Delta \Delta \mathrm{G}$ implies destabilization while the negative change implies stabilization of the variant with respect to the WT. The error bars are shown. 


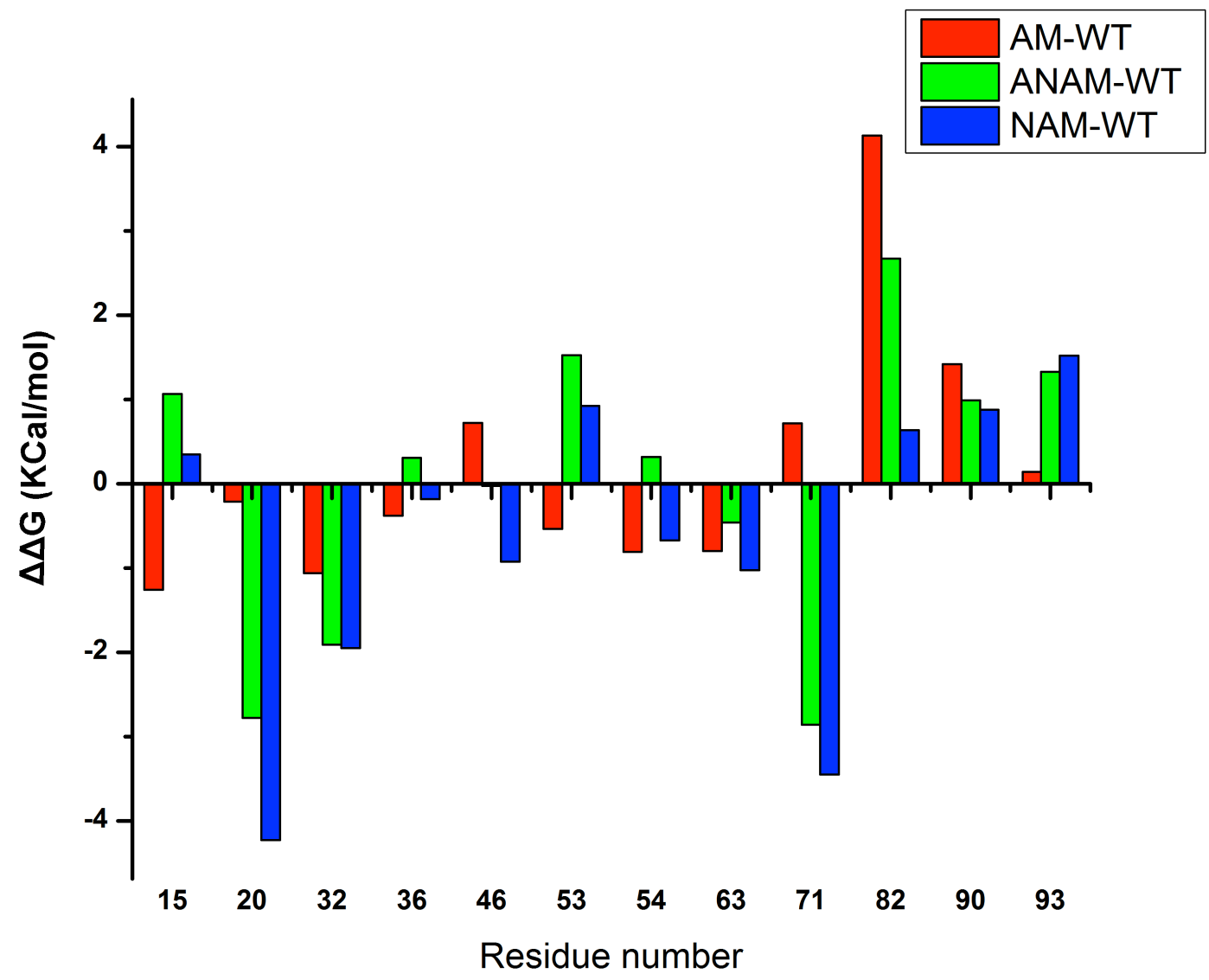

Figure S5: Residue-level decomposition of $\Delta \Delta \mathrm{G}^{\mathrm{F}}$ for the protease variants. Contributions are shown for those select residues, which were mutated in the drug-resistant isolate under study, CA84179. 


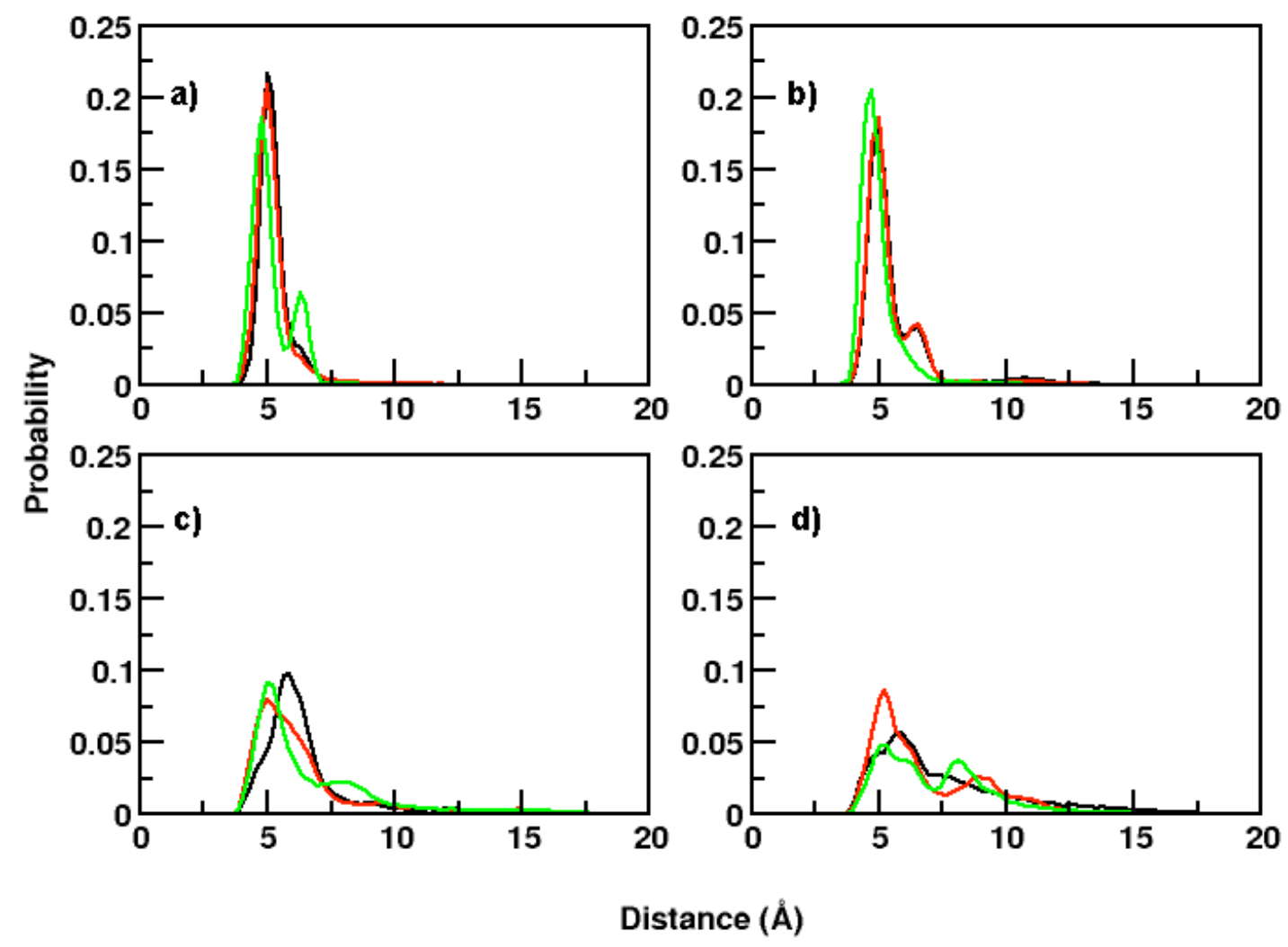

Figure S6: Flap tip -flap tip distances of a) WT, b) AM, c) ANAM and d) NAM proteases show well-converged probability distributions during the three independent $100 \mathrm{~ns}$ MD simulations, as depicted in black, red, and green curves. 


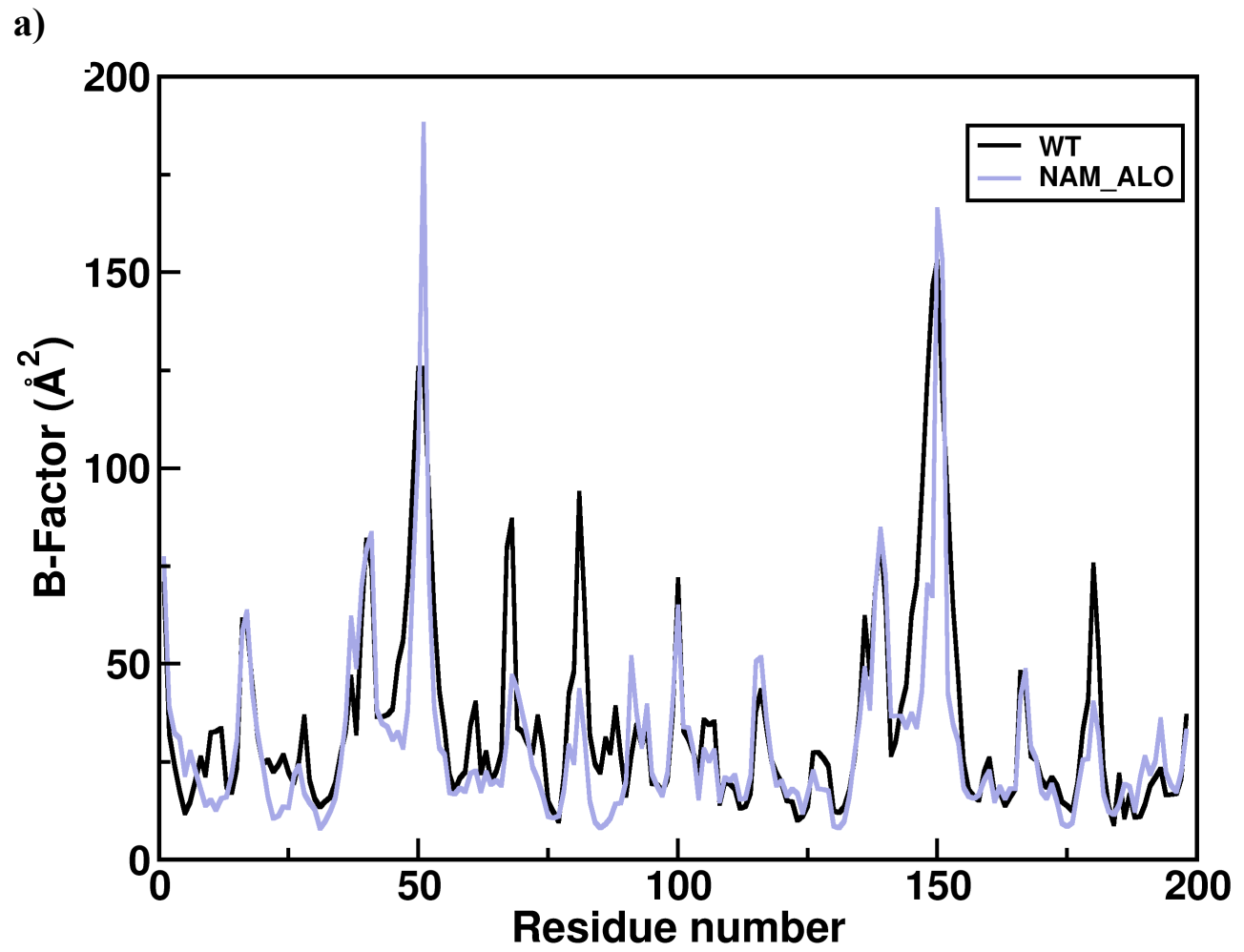

b)

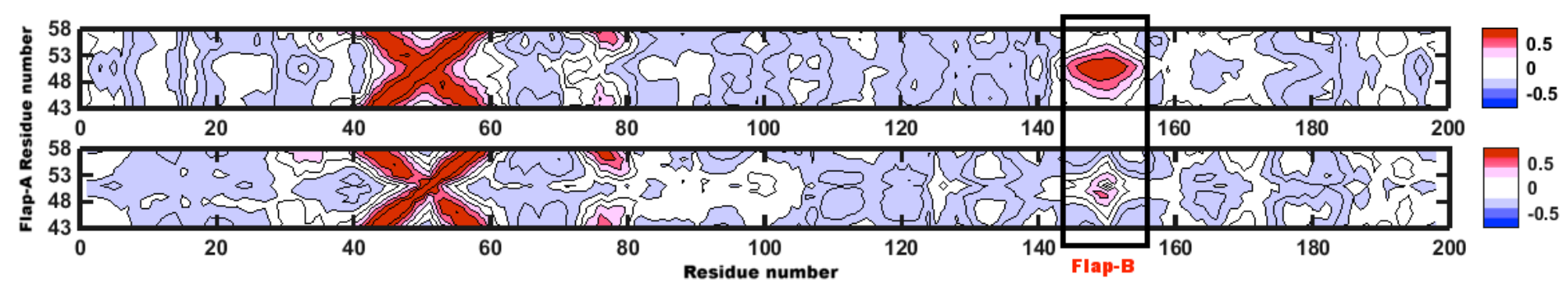

Figure S7: Effect of distal non-active site mutations alone on protease dynamics. (a) Average Bfactor of each protease residues in WT and NAM_ALO. The color code is shown as inset. (b) Dynamic cross-correlation map of flap-A residues with all protein residues in WT (first row) and NAM_ALO (second row). 
Table S2: Mutational prevalence of the residues of HIV-1 protease in naive and protease inhibitor (PI) treated HIV patients, as obtained from HIV drug resistance database (hivdb.stanford.edu). Residues showing $<=1 \%$ of mutational threshold are highlighted in green (conserved), while residues having mutational threshold between 1 to $10 \%$ are highlighted in blue. Highly mutated residues (having $>=10 \%$ of mutational threshold) are highlighted in orange.

In column 6 , prediction of conserved-ness of the residues is tabulated based on CPL analysis. Residues are predicted to be conserved if Z-score of $\Delta \mathrm{CPL}$ were $>=0.68$ and confidence interval $>=75 \%$. Those conserved residues are designated "Yes" in column 6. Else they are designated "No". Accuracy of CPL-based prediction is calculated from the ratio of number of correct predictions (i.e. true positives + true negatives) and total number of nonperipheral nodes in protease network. Non-peripheral nodes are defined by nodes having degree $>3$ (column 7). Definition of true positive: green row with CPL prediction "Yes"; true negative: orange row with CPL prediction "No".

\begin{tabular}{|c|c|c|c|c|c|c|}
\hline $\begin{array}{l}\text { Residue } \\
\text { No. of } \\
\text { HIV-1 } \\
\text { Protease }\end{array}$ & $\begin{array}{l}\% \\
\text { mutations } \\
\text { in naive } \\
\text { HIV } \\
\text { patients } \\
\text { (out of } \\
\text { total } \\
27670 \\
\text { isolates of } \\
\text { subtype } \\
\text { B) }\end{array}$ & $\begin{array}{l}\% \\
\text { mutations } \\
\text { in PI } \\
\text { treated } \\
\text { HIV } \\
\text { patients } \\
\text { (out of } \\
\text { total 9554 } \\
\text { isolates of } \\
\text { subtype B) }\end{array}$ & $\begin{array}{l}\% \\
\text { mutatios } \\
\text { in naive } \\
\text { HIV } \\
\text { patients } \\
\text { (out of } \\
\text { total } \\
58521 \\
\text { isolates } \\
\text { of all } \\
\text { subtypes) }\end{array}$ & $\begin{array}{l}\text { \% } \\
\text { mutations in } \\
\text { PI treated } \\
\text { HIV } \\
\text { patients (out } \\
\text { of total } \\
13705 \\
\text { isolates of } \\
\text { all } \\
\text { subtypes) }\end{array}$ & $\begin{array}{l}\text { Prediction of } \\
\text { conserved } \\
\text { residues from } \\
\text { CPL analysis }\end{array}$ & $\begin{array}{l}\text { Number of } \\
\text { links of a } \\
\text { node with } \\
\text { other nodes } \\
\text { in the } \\
\text { network } \\
\text { (degree) }\end{array}$ \\
\hline 1 & 0 & 0 & 0 & 0 & $\mathrm{No}$ & 2 \\
\hline 2 & 0.1 & 0.1 & 0 & 0.1 & No & 4 \\
\hline 3 & 0.5 & 0.1 & 0.4 & 0.1 & $\mathrm{No}$ & 4 \\
\hline 4 & 0.4 & 0.7 & 0.3 & 0.6 & No & 1 \\
\hline 5 & 0.1 & 0.1 & 0.1 & 0.1 & No & 8 \\
\hline
\end{tabular}




\begin{tabular}{|c|c|c|c|c|c|c|}
\hline 6 & 0.1 & 0.2 & 0.1 & 0.3 & No & 2 \\
\hline 7 & 0.2 & 0.3 & 0.3 & 0.3 & No & 1 \\
\hline 8 & 0 & 0.2 & 0 & 0.2 & Yes & 5 \\
\hline 9 & 0 & 0 & 0 & 0 & No & 7 \\
\hline 10 & 10.7 & 55.5 & 11.7 & 50.4 & No & 4 \\
\hline 11 & 0.5 & 3.6 & 0.7 & 2.9 & No & 7 \\
\hline 12 & 17.2 & 12.4 & 22.8 & 16.6 & No & 6 \\
\hline 13 & 14.2 & 25.4 & 35.4 & 27.6 & No & 9 \\
\hline 14 & 12.2 & 8.6 & 19.4 & 11.5 & No & 4 \\
\hline 15 하 & 20.7 & 20.3 & 30.3 & 33.4 & No & 8 \\
\hline 16 & 4.4 & 6.3 & 10.4 & 8.4 & No & 3 \\
\hline 17 & 0.9 & 0.9 & 2.4 & 1.6 & No & 0 \\
\hline 18 & 1.7 & 2.7 & 1.4 & 2.4 & No & 2 \\
\hline 19 & 11.8 & 14.3 & 23.8 & 18.7 & No & 3 \\
\hline 20 ฟ & 2.4 & 28.4 & 20.3 & 36.1 & No & 5 \\
\hline 21 & 0.1 & 0.1 & 0.1 & 0.3 & No & 5 \\
\hline 22 & 0 & 0.7 & 0 & 0.8 & No & 5 \\
\hline 23 & 0 & 1 & 0 & 0.9 & Yes & 9 \\
\hline 24 & 0 & 7.7 & 0 & 6.5 & No & 11 \\
\hline 25 & 0 & 0 & 0 & 0 & Yes & 7 \\
\hline 26 & 0 & 0 & 0 & 0 & Yes & 9 \\
\hline 27 & 0 & 0 & 0 & 0 & No & 3 \\
\hline 28 & 0 & 0 & 0 & 0 & No & 4 \\
\hline 29 & 0 & 0 & 0 & 0 & Yes & 4 \\
\hline
\end{tabular}




\begin{tabular}{|c|c|c|c|c|c|c|}
\hline 30 & 0 & 7.1 & 0 & 6 & No & 4 \\
\hline 31 & 0 & 0 & 0 & 0 & Yes & 7 \\
\hline 32 ț & 0 & 6.6 & 0 & 4.8 & No & 5 \\
\hline 33 & 3.3 & 17.6 & 2.3 & 14.3 & No & 10 \\
\hline 34 & 0.1 & 4.2 & 0.4 & 3.2 & No & 7 \\
\hline 35 & 30.4 & 33.1 & 43.2 & 44.3 & No & 4 \\
\hline 36 ฝు & 16.6 & 37.4 & 58.5 & 55.8 & No & 6 \\
\hline 37 & 36.4 & 35.7 & 27 & 31.2 & No & 1 \\
\hline 38 & 0.2 & 0.4 & 0.3 & 0.2 & No & 6 \\
\hline 39 & 3.9 & 2.1 & 3.4 & 2.5 & No & 3 \\
\hline 40 & 0 & 0 & 0 & 0 & No & 1 \\
\hline 41 & 32.4 & 21.2 & 64.8 & 42.7 & No & 2 \\
\hline 42 & 0.1 & 0.1 & 0.1 & 0 & No & 4 \\
\hline 43 & 2.5 & 7.7 & 2.1 & 7.2 & No & 2 \\
\hline 44 & 0 & 0 & 0 & 0 & No & 3 \\
\hline 45 & 1.3 & 2.2 & 3 & 3 & No & 4 \\
\hline $46 \quad \hbar$ & 0.5 & 37.6 & 0.5 & 31.1 & No & 4 \\
\hline 47 & 0 & 6.1 & 0 & 4.8 & No & 5 \\
\hline 48 & 0 & 5.9 & 0 & 4.9 & No & 4 \\
\hline 49 & 0 & 0 & 0 & 0 & No & 3 \\
\hline 50 & 0 & 2.5 & 0 & 2.3 & No & 7 \\
\hline 51 & 0 & 0.3 & 0 & 0.2 & No & 3 \\
\hline 52 & 0 & 0 & 0 & 0 & No & 3 \\
\hline 53 & 0 & 6 & 0 & 5.4 & No & 5 \\
\hline 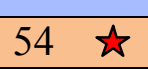 & 0 & 37.8 & 0 & 33.5 & No & 6 \\
\hline
\end{tabular}




\begin{tabular}{|c|c|c|c|c|c|c|}
\hline 55 & 0.2 & 7.2 & 0.2 & 6.6 & No & 3 \\
\hline 56 & 0 & 0 & 0 & 0 & No & 8 \\
\hline 57 & 11.7 & 12.3 & 14 & 22.6 & No & 8 \\
\hline 58 & 0.3 & 6.9 & \begin{tabular}{|l|} 
\\
\end{tabular} & 5.7 & No & 6 \\
\hline 59 & $\mid 0.1$ & 0 & \begin{tabular}{|l|l}
0.1 \\
\end{tabular} & 0 & Yes & 11 \\
\hline 60 & 9.3 & 10.7 & 9.2 & 11.4 & No & 4 \\
\hline 61 & \begin{tabular}{|l|l}
4.7 \\
\end{tabular} & 6.4 & 6.6 & \begin{tabular}{|l|l}
18.8 \\
\end{tabular} & No & 3 \\
\hline 62 & 24.2 & 38.2 & \begin{tabular}{|l|l}
13.7 \\
\end{tabular} & 30.6 & No & 6 \\
\hline $63 \leftarrow$ & 82.5 & 89.3 & 62 & 79.7 & No & 4 \\
\hline 64 & 18.9 & 22 & 13.7 & 17.8 & No & 7 \\
\hline 65 & 3.2 & 1.8 & 2.9 & 3.2 & No & 5 \\
\hline 66 & 0 & 2.8 & \begin{tabular}{|l|}
0.1 \\
\end{tabular} & 2.4 & No & 8 \\
\hline 67 & 2.9 & 3.6 & \begin{tabular}{|l|l|}
4.1 \\
\end{tabular} & 3.6 & No & 4 \\
\hline 68 & 0.6 & 0.4 & 0.6 & 0.6 & No & 0 \\
\hline 69 & \begin{tabular}{|l}
7.7 \\
\end{tabular} & 9 & 52.5 & 23.3 & No & 4 \\
\hline 70 & 5.9 & 3.4 & 8.6 & 4.4 & No & 5 \\
\hline 71 كֵ & 13.2 & 47.9 & 6.6 & 38 & No & 6 \\
\hline 72 & 16.5 & 21.3 & 10.9 & 20.5 & No & 6 \\
\hline 73 & 0 & 15.2 & 0 & $\begin{array}{ll}1.3 \\
\end{array}$ & No & 6 \\
\hline 74 & 0.8 & 8.1 & 2.9 & 10.2 & No & 6 \\
\hline 75 & 0.3 & 0.6 & 0.3 & 0.6 & Yes & 8 \\
\hline 76 & 0 & 3.9 & 0 & 3.7 & Yes & 10 \\
\hline 77 & 30.9 & 29.6 & \begin{tabular}{|l|}
17.8 \\
\end{tabular} & 22.1 & No & 8 \\
\hline 78 & 0 & 0 & 0 & 0 & No & 5 \\
\hline
\end{tabular}




\begin{tabular}{|c|c|c|c|c|c|c|}
\hline 79 & 0.5 & 1.9 & 0.3 & 1.4 & No & 4 \\
\hline 80 & 0 & 0 & 0 & 0 & No & 4 \\
\hline 81 & 0 & 0 & 0 & 0 & No & 3 \\
\hline 82 స & 1.7 & 34.7 & 5.3 & 33.9 & No & 2 \\
\hline 83 & 0 & 0.8 & 0 & 0.9 & Yes & 6 \\
\hline 84 & 0 & 18.1 & 0 & 14 & No & 8 \\
\hline 85 & 0.1 & 4.6 & 0.1 & 3.9 & Yes & 11 \\
\hline 86 & 0 & 0 & 0 & 0 & No & 6 \\
\hline 87 & 0.1 & 0.1 & 0 & 0 & Yes & 8 \\
\hline 88 & 0 & 6.7 & 0 & 6.2 & No & 3 \\
\hline 89 & 1.7 & 6.6 & \begin{tabular}{|l|}
46.2 \\
\end{tabular} & 21.2 & No & 10 \\
\hline 90 추 & \begin{tabular}{|l|}
0.4 \\
\end{tabular} & 38.4 & 0.3 & 31.7 & Yes & 9 \\
\hline 91 & 0.3 & 1.8 & 0.4 & 1.7 & No & 4 \\
\hline 92 & \begin{tabular}{|l|}
1.4 \\
\end{tabular} & 4.3 & \begin{tabular}{|l|}
0.9 \\
\end{tabular} & 3.5 & No & 6 \\
\hline 93 & \begin{tabular}{|l|}
43 \\
\end{tabular} & 39.8 & \begin{tabular}{|l|}
42.9 \\
\end{tabular} & 42.4 & No & 8 \\
\hline 94 & 0 & 0 & 0 & 0 & No & 2 \\
\hline 95 & 0.4 & 2.4 & 0.2 & 1.9 & No & 7 \\
\hline 96 & 0 & 0.3 & \begin{tabular}{|l|}
0.1 \\
\end{tabular} & 0.3 & No & 6 \\
\hline 97 & 0 & 0 & \begin{tabular}{|l|}
0.1 \\
\end{tabular} & 0 & No & 10 \\
\hline 98 & 0.2 & 0 & 0.2 & 0.1 & No & 4 \\
\hline 99 & 0 & 0.2 & 0 & 0 & No & 8 \\
\hline Accuracy & & & & & $73 \%$ & \\
\hline
\end{tabular}

Indicates mutations present in the isolate under study. 
Table S3: List of all protease residues that positively interfere with the centrality of active site and flap residues

List of residues that increase the centrality of active site residues, L23-G27

List of residues that increase the centrality of flap residues, M46-I54
Local: R8 - V11, A22, A28-L33, V82-L90, I93, C95, R107-V110, A121 - G126, D129 - L132, E165

Distal: K20, M36, I50, R57, Q58, Y59, K70L76, T80, Q92, I112, E164, L175, T179.

Local: P44-Q58, L76, V77, P79-V82, I84, G147-F152, L178-V181, I183

Distal: P9, V11, I15, K20, L23, E34, M36, K43, V110, E120, L123, E133, Y158, N182 
Table S4: Optimal and suboptimal paths for communications of the mutated residues to the central hubs in functional regions (target residues). Results are shown for the WT and NAM variant of proteases and for the top five shortest path distances between each pair of mutated and target residues.

\begin{tabular}{|c|c|c|c|c|}
\hline $\begin{array}{l}\text { Mutated } \\
\text { residue }\end{array}$ & $\begin{array}{l}\text { Target } \\
\text { residue }\end{array}$ & $\begin{array}{l}\text { Optimal and Suboptimal paths in } \\
\text { WT }\end{array}$ & $\begin{array}{l}\text { Optimal and Suboptimal paths in } \\
\text { NAM }\end{array}$ & $\begin{array}{l}\text { Change } \\
\text { in path } \\
\text { distance } \\
\text { (WT- } \\
\text { NAM) } \\
\text { from } \\
\text { top five } \\
\text { paths }\end{array}$ \\
\hline 15 & 50 & $\begin{array}{l}\text { I15-K20-E34-P81-I50 (9.75) } \\
\left(\mathrm{OP}{ }_{\mathrm{md} 1} ; \mathrm{OP}_{\mathrm{md} 2} ; \mathrm{SOP} 1_{\mathrm{md} 3}\right) \\
\mathrm{I} 15-\mathrm{L} 33-\mathrm{I} 84-\mathrm{V} 82-\mathrm{I} 50(10.54) \\
\left(\mathrm{SOP} 1_{\mathrm{md} 1} ; \mathrm{SOP} 1_{\mathrm{md} 2} ; \mathrm{OP}_{\mathrm{md} 3}\right) \\
\mathrm{I} 15-\mathrm{K} 20-\mathrm{E} 34-\mathrm{T} 80-\mathrm{V} 82-\mathrm{I} 50 \\
(10.56)\left(\mathrm{SOP} 3_{\mathrm{md} 1} ; \mathrm{SOP} 2_{\mathrm{md} 2} ;\right. \\
\left.\mathrm{SOP} 2_{\mathrm{md} 3}\right) \\
\text { I15-Q18-K20-E34-T80-V82-I50 } \\
(10.68)\left(\mathrm{SOP} 2_{\mathrm{md} 1} ; \mathrm{SOP} 3_{\mathrm{md} 2} ;\right. \\
\left.\text { SOP3 }{ }_{\mathrm{md} 3}\right) \\
\text { I15-L33-N83-E34-P81-I50 } \\
(11.17)\left(\mathrm{SOP} 4_{\mathrm{md} 1} ; \mathrm{SOP} 7_{\mathrm{md} 2} ;\right. \\
\left.\text { SOP5 }{ }_{\mathrm{md} 3}\right)\end{array}$ & $\begin{array}{l}\text { V15-L33-L76-I47-I54-G52-I50 } \\
(14.82)\left(\mathrm{OP} \mathrm{md}_{1} ; \mathrm{SOP} 2_{\mathrm{md} 2} ;\right. \\
\left.\mathrm{SOP} 1_{\mathrm{md} 3}\right) \\
\text { V15-L33-V77-V56-I47-I54- } \\
\text { G52-I50 (14.93) (SOP1 }{ }_{\mathrm{md} 1} ; \\
\left.\text { SOP3 }{ }_{\mathrm{md} 2} ; \mathrm{OP}_{\mathrm{md} 3}\right) \\
\text { V15-I36-V77-V56-I54-G52-I50 } \\
(15.28)\left(\mathrm{SOP} 2_{\mathrm{md} 1} ; \mathrm{SOP} 4_{\mathrm{md} 2} ;\right. \\
\left.\text { SOP3 }{ }_{\mathrm{md} 3}\right) \\
\text { V15-L33-L76-I32-I47-I54-G52- } \\
\text { I50 (15.59) (SOP3 }{ }_{\mathrm{md} 1} ; \mathrm{OP}_{\mathrm{md} 2} ; \\
\left.\text { SOP6 } 6_{\mathrm{md} 3}\right) \\
\text { V15-Q18-R20-E34-V77-V56- } \\
\text { I54-G52-I50 (15.84) (SOP4 }{ }_{\mathrm{md} 1} \text {; } \\
\left.\text { SOP1 } 1_{\mathrm{md} 2} ; \mathrm{SOP} 2_{\mathrm{md} 3}\right)\end{array}$ & -4.75 \\
\hline 20 & 50 & $\begin{array}{l}\text { K20-E34-P81-I50 (7.68) }\left(\mathrm{OP}_{\mathrm{md} 1} ;\right. \\
\left.\mathrm{OP}_{\mathrm{md} 2} ; \mathrm{SOP} 1_{\mathrm{md} 3}\right) \\
\mathrm{K} 20-\mathrm{E} 34-\mathrm{T} 80-\mathrm{V} 82-\mathrm{I} 50(8.67) \\
\left(\mathrm{SOP} 1_{\mathrm{md} 1} ; \mathrm{SOP} 1_{\mathrm{md} 2} ; \mathrm{OP} \mathrm{md}_{3}\right) \\
\mathrm{K} 20-\mathrm{E} 34-\mathrm{N} 83-\mathrm{T} 80-\mathrm{V} 82-\mathrm{I} 50 \\
(9.23)\left(\mathrm{SOP} 2_{\mathrm{md} 1} ; \mathrm{SOP} 3_{\mathrm{md} 2} ;\right.\end{array}$ & $\begin{array}{l}\text { R20-E35-R57-L76-I47-I54-G52- } \\
\text { II50 (13.03) }\left(\mathrm{OP}_{\mathrm{md} 1} ; \mathrm{SOP} 4_{\mathrm{md} 2}\right. \\
\left.\mathrm{SOP} 3_{\mathrm{md} 3}\right) \\
\text { R20-E35-R57-V77-E34-P81-I50 } \\
(13.15)\left(\mathrm{SOP} 1_{\mathrm{md} 1} ; \mathrm{SOP} 5_{\mathrm{md} 2} ;\right. \\
\left.\mathrm{OP}_{\mathrm{md} 3}\right)\end{array}$ & -4.61 \\
\hline
\end{tabular}




\begin{tabular}{|c|c|c|c|c|}
\hline & & $\begin{array}{l}\left.\mathrm{SOP} 3_{\mathrm{md} 3}\right) \\
\text { K20-E34-G78-T80-V82-I50 } \\
(9.36)\left(\mathrm{SOP} 3_{\mathrm{md} 1} ; \mathrm{SOP} 2_{\mathrm{md} 2} ;\right. \\
\left.\mathrm{SOP} 2_{\mathrm{md} 3}\right) \\
\text { K20-E34-N83-V32-I84-V82-I50 } \\
(9.56)\left(\mathrm{SOP} 4_{\mathrm{md} 1} ; \mathrm{SOP} 4_{\mathrm{md} 2} ;\right. \\
\left.\mathrm{SOP} 4_{\mathrm{md} 3}\right)\end{array}$ & $\begin{array}{l}\text { R20-E35-G78-V56-I54-G52-I50 } \\
(13.52)\left(\mathrm{SOP} 2_{\mathrm{md} 1} ; \mathrm{SOP}_{\mathrm{md} 2} ;\right. \\
\left.\mathrm{SOP} 6_{\mathrm{md} 3}\right) \\
\text { R20-E34-V77-V56-I54-G52-I50 } \\
(13.92)\left(\mathrm{SOP} 3_{\mathrm{md1}} ; \mathrm{OP}_{\mathrm{md} 2} ;\right. \\
\left.\mathrm{SOP} 5_{\mathrm{md} 3}\right) \\
\text { R20-E34-N83-I32-I47-I54-G52- } \\
\text { I50 (13.96) (SOP4 }{ }_{\mathrm{md} 1} ; \mathrm{SOP} 2_{\mathrm{md} 2} ; \\
\left.\mathrm{SOP} 2_{\mathrm{md} 3}\right)\end{array}$ & \\
\hline 36 & 50 & 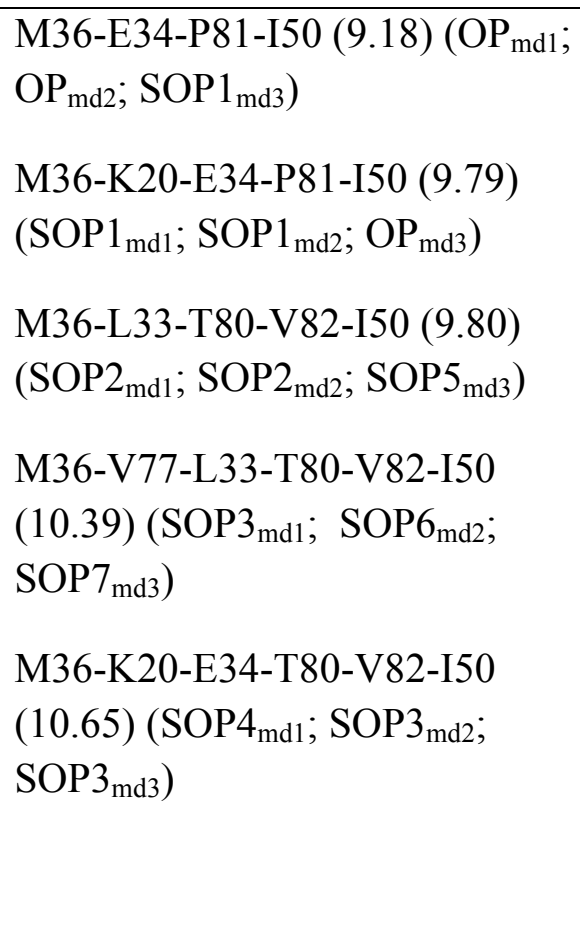 & $\begin{array}{l}\text { I36-V77-V56-I54-G52-I50 } \\
(12.14)\left(\mathrm{OP}{ }_{\mathrm{md} 1} ; \mathrm{OP}_{\mathrm{md} 2} ; \mathrm{SOP} 2_{\mathrm{md} 3}\right) \\
\text { I36-V77-V56-I47-I54-G52-I50 } \\
(13.74) \mathrm{SOP} 1_{\mathrm{md} 1} ; \mathrm{SOP} 2_{\mathrm{md} 2} ; \\
\left.\mathrm{SOP}_{\mathrm{md} 3}\right) \\
\text { I36-R57-V77-V56-I54-G52-I50 } \\
(14.47)\left(\mathrm{SOP} 2_{\mathrm{md} 1} ; \mathrm{SOP} 6_{\mathrm{md} 2} ;\right. \\
\left.\mathrm{SOP} 3_{\mathrm{md} 3}\right) \\
\mathrm{I} 36-\mathrm{E} 34-\mathrm{V} 77-\mathrm{V} 56-\mathrm{I} 54-\mathrm{G} 52-\mathrm{I} 50 \\
(14.50)\left(\mathrm{SOP} 3_{\mathrm{md} 1} ; \mathrm{SOP} 7_{\mathrm{md} 2} ;\right. \\
\left.\mathrm{SOP} 7_{\mathrm{md} 3}\right) \\
\mathrm{I} 36-\mathrm{V} 77-\mathrm{V} 56-\mathrm{I} 47-\mathrm{G} 49-\mathrm{G} 52-\mathrm{I} 50 \\
(14.75)\left(\mathrm{SOP} 4_{\mathrm{md} 1} ; \mathrm{SOP} 5_{\mathrm{md} 2} ;\right. \\
\left.\mathrm{SOP} 9_{\mathrm{md} 3}\right)\end{array}$ & -3.96 \\
\hline 71 & 24 & $\begin{array}{l}\text { A71-I93-L24 (6.92) }\left(\mathrm{OP}_{\mathrm{md} 1} ;\right. \\
\left.\mathrm{OP}_{\mathrm{md} 2} ; \mathrm{SOP} 1_{\mathrm{md} 3}\right) \\
\text { A71-I64-I85-L24 (7.01) } \\
\left(\mathrm{SOP} 1_{\mathrm{md} 1} ; \mathrm{SOP} 1_{\mathrm{md} 2} ; \mathrm{OP}{ }_{\mathrm{md}}\right) \\
\text { A71-I93-L90-L24 (7.18) } \\
\left(\mathrm{SOP} 2_{\mathrm{md} 1} ; \mathrm{SOP} 2_{\mathrm{md} 2} ; \mathrm{SOP} 2_{\mathrm{md} 3}\right) \\
\text { A71-L89-I85-L24 (7.54) } \\
\left(\mathrm{SOP} 3_{\mathrm{md} 1} ; \mathrm{SOP} 3_{\mathrm{md} 2} ; \mathrm{SOP} 3_{\mathrm{md} 3}\right) \\
\text { A71-Q92-L90-L24 (7.73) }\end{array}$ & $\begin{array}{l}\text { I71-L93-L24 (5.67) }\left(\mathrm{OP}_{\mathrm{md} 1} ;\right. \\
\left.\mathrm{OP}_{\mathrm{md} 2} ; \mathrm{OP}_{\mathrm{md} 3}\right) \\
\text { I71-L93-M90-L24 (6.14) } \\
\left(\mathrm{SOP} 1_{\mathrm{md} 1} ; \mathrm{SOP} 1_{\mathrm{md} 2} ; \mathrm{SOP} 1_{\mathrm{md} 3}\right) \\
\mathrm{I} 71-\mathrm{L} 93-198-\mathrm{L} 24(6.61) \\
\left(\mathrm{SOP} 2_{\mathrm{md} 1} ; \mathrm{SOP} 3_{\mathrm{md} 2} ; \mathrm{SOP} 2_{\mathrm{md} 3}\right) \\
\text { I71-I66-I85-L24 (6.68) } \\
\left(\mathrm{SOP} 3_{\mathrm{md} 1} ; \mathrm{SOP} 2_{\mathrm{md} 2} ; \mathrm{SOP} 3_{\mathrm{md} 3}\right) \\
\text { I71-L89-I85-L24 (6.69) }\end{array}$ & 0.88 \\
\hline
\end{tabular}




\begin{tabular}{|c|c|c|c|c|}
\hline & & $\left(\mathrm{SOP} 4_{\mathrm{md} 1} ; \mathrm{SOP} 4_{\mathrm{md} 2} ; \mathrm{SOP} 4_{\mathrm{md} 3}\right)$ & $\left(\mathrm{SOP} 5_{\mathrm{md} 1} ; \mathrm{SOP} 4_{\mathrm{md} 2} ; \mathrm{SOP} 4_{\mathrm{md} 3}\right)$ & \\
\hline 90 & 26 & $\begin{array}{l}\mathrm{L} 90-\mathrm{T} 26(3.00)\left(\mathrm{OP}_{\mathrm{md} 1} ; \mathrm{OP}_{\mathrm{md} 2} ;\right. \\
\left.\mathrm{OP}_{\mathrm{md} 3}\right) \\
\mathrm{L} 90-\mathrm{R} 87-\mathrm{T} 26(4.85)\left(\mathrm{SOP} 1_{\mathrm{md} 1}\right. \\
\left.\mathrm{SOP} 2_{\mathrm{md} 2} ; \mathrm{SOP} 1_{\mathrm{md} 3}\right) \\
\mathrm{L} 90-\mathrm{L} 104-\mathrm{T} 26(4.77)\left(\mathrm{SOP} 2_{\mathrm{md} 1}\right. \\
\left.\mathrm{SOP} 1_{\mathrm{md} 2} ; \mathrm{SOP} 2_{\mathrm{md} 3}\right) \\
\text { L90-R87-L104-T26 (5.59) } \\
\left(\mathrm{SOP} 4_{\mathrm{md} 1} ; \mathrm{SOP} 3_{\mathrm{md} 2} ; \mathrm{SOP} 3_{\mathrm{md} 3}\right) \\
\mathrm{L}{ }-\mathrm{D} 25-\mathrm{D} 124-\mathrm{T} 26(5.75) \\
\left(\mathrm{SOP} 3_{\mathrm{md} 1} ; \mathrm{SOP} 4_{\mathrm{md} 2} ; \mathrm{SOP} 4_{\mathrm{md} 3}\right)\end{array}$ & 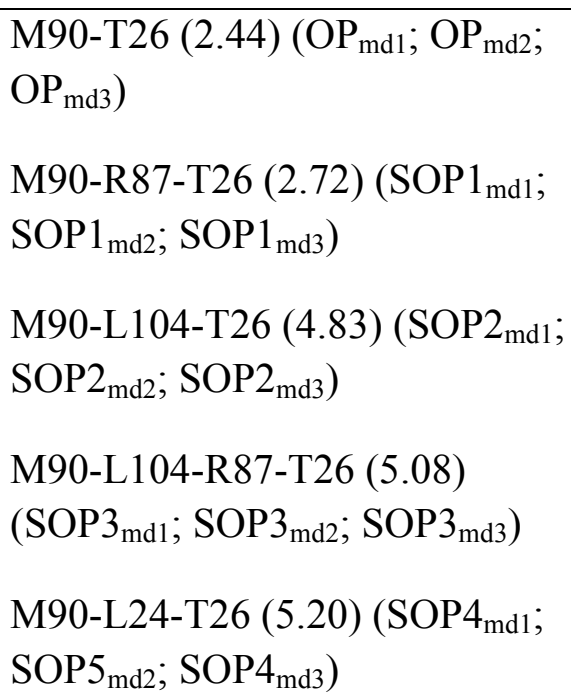 & 0.74 \\
\hline 93 & 24 & $\begin{array}{l}\text { I93-L24 (4.06) }\left(\mathrm{OP}_{\mathrm{md} 1} ; \mathrm{OP}_{\mathrm{md} 2}\right. \\
\left.\mathrm{OP}_{\mathrm{md} 3}\right) \\
\mathrm{I} 93-\mathrm{L} 90-\mathrm{L} 24(4.25)\left(\mathrm{SOP} 1_{\mathrm{md} 1}\right. \\
\left.\mathrm{SOP} 1_{\mathrm{md} 2} ; \mathrm{SOP} 1_{\mathrm{md} 3}\right) \\
\mathrm{I} 93-\mathrm{F} 198-\mathrm{L} 24(4.98)\left(\mathrm{SOP} 2_{\mathrm{md} 1}\right. \\
\left.\mathrm{SOP} 2_{\mathrm{md} 2} ; \mathrm{SOP} 2_{\mathrm{md} 3}\right) \\
\mathrm{I} 93-\mathrm{L} 90-\mathrm{I} 85-\mathrm{L} 24(5.69) \\
\left(\mathrm{SOP} 3_{\mathrm{md} 1} ; \mathrm{SOP} 3_{\mathrm{md} 2} ; \mathrm{SOP} 3_{\mathrm{md} 3}\right) \\
\mathrm{I} 93-\mathrm{I} 66-\mathrm{I} 85-\mathrm{L} 24(6.69) \\
\left(\mathrm{SOP} 4_{\mathrm{md} 1} ; \mathrm{SOP} 4_{\mathrm{md} 2} ; \mathrm{SOP} 4_{\mathrm{md} 3}\right)\end{array}$ & 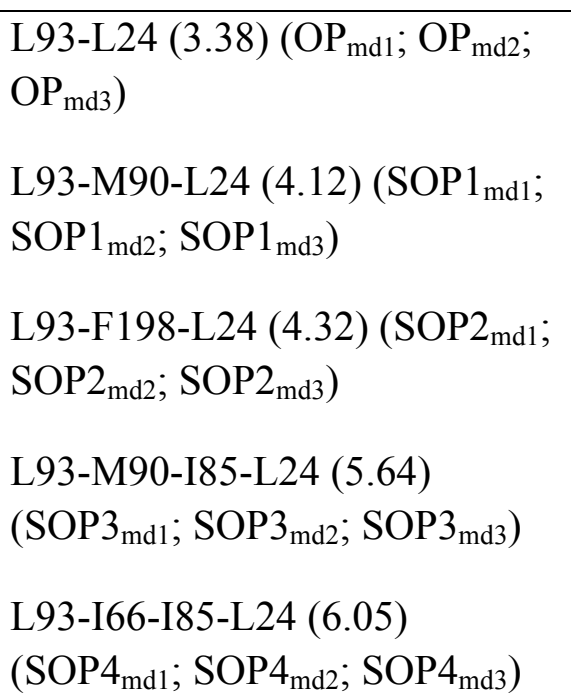 & 0.43 \\
\hline
\end{tabular}

OP stand for optimal path, SOP1 stands for suboptimal path 1, SOP2 stand for suboptimal path 2, SOP3 stand for suboptimal path 3, SOP4 stand for suboptimal path 4, md1 stands for network analysis data from MD simulation 1, md2 stands for network analysis data from MD simulation 2, md3 stands for network analysis data from MD simulation 3. 
Table S5: Free energy decomposition to obtain the free energy of individual monomer and monomer-monomer interface energy in WT and protease variants.

\begin{tabular}{|l|c|l|l|c|}
\hline $\begin{array}{l}\text { HIV-1 } \\
\text { protease } \\
\text { type }\end{array}$ & $\begin{array}{l}\text { Free energy } \\
\text { of the dimer } \\
\left(\mathrm{G}_{\mathrm{d}} \text { in }\right. \\
\mathrm{kcal} / \mathrm{mol})\end{array}$ & $\begin{array}{l}\text { Free energy } \\
\text { of } \\
\text { monomer-A } \\
\left(\mathrm{G}_{\mathrm{mA}} \text { in }\right. \\
\mathrm{kcal} / \mathrm{mol})\end{array}$ & $\begin{array}{l}\text { Free energy } \\
\text { of } \\
\text { monomer-B } \\
\left(\mathrm{G}_{\mathrm{mB}} \text { in }\right. \\
\mathrm{kcal} / \mathrm{mol})\end{array}$ & $\begin{array}{l}\Delta \mathrm{G}\left(\mathrm{G}_{\mathrm{d}}-\right. \\
\mathrm{G}_{\mathrm{mA}}-\mathrm{G}_{\mathrm{mB}} \\
\text { in kcal/mol })\end{array}$ \\
\hline WT & -3870 & -1921 & -1921 & -28 \\
\hline AM & -3787 & -1877 & -1877 & -33 \\
\hline ANAM & -4159 & -2069 & -2069 & -21 \\
\hline NAM & -4247 & -2110 & -2110 & -27 \\
\hline
\end{tabular}




\section{SI Text. S1: In the context of "Mutations repartition the protease network community"}

To start with, we attempted to extract the community structure of HIV-1 protease using the wellknown Louvain algorithm (Ref-31 in main text). This algorithm relies upon the principle of maximizing the network modularity by optimal repartitioning of the nodes. Figure S8 shows the community structures of the WT and NAM protease, as obtained by utilizing this algorithm. While the WT protease network was partitioned into seven communities, the NAM network exhibited nine distinct communities. More importantly, while the inter-flap residues belonged to the same community in WT, they were partitioned to different communities in NAM. This signifies the uncorrelated movements of the flaps in NAM variants, which again in excellent agreement with this and other MD reports. Moreover, the computed modularity values (which signifies the evolvability and adaptability of the system to new environments ${ }^{1}$ ) show that the NAM and ANAM have higher modularity index than the WT; and AM has the least modularity (Table S6). This implies an increased adaptability of the ANAM variant to drug pressure, while the AM is more vulnerable than even the WT. Hence for sustainability, AM seeks non-active site mutations and the AM+NAM i.e. the ANAM variant of HIV protease is produced that resist the drugs profoundly. Our hypothesis is very well supported by the fact that the yeast protein interaction network exhibits a considerable increase in modularity, concurrent with its significant topological differences along evolution (adaptability to change of environment). ${ }^{2}$

Even though the community structure due to Louvain algorithm provides a good description of protease's modularity, it shows a large amount of information loss in mutants relative to the WT due to large variation of information $\left(\mathrm{V}_{\mathrm{IN}}\right)$ or node repartitioning. The repartitioning of inter-flap residues in Fig. S8 is one such example. The total $V_{I N}$ in each of the variant with respect to the WT is tabulated in Table S6. A large variation of $30 \%$ indicates a dissimilar community 
structure, which makes the comparison of networks difficult. Moreover, it does not demarcate the domains and functional regions of HIV protease explicitly. 

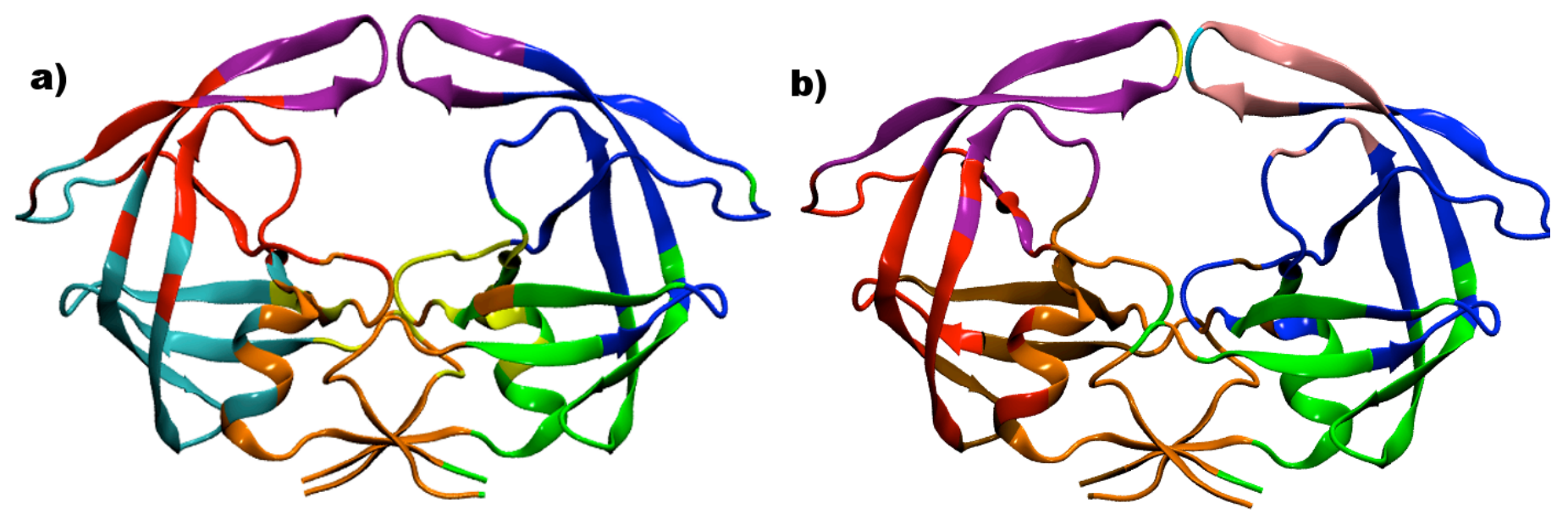

Figure S8: Community structure of (a) WT and (b) NAM variant of HIV-1 proteases as obtained through Louvain modularity maximization approach. While Louvain algorithm partitions WT protease network into seven communities (depicted by seven individual colors), the NAM network was partitioned into nine distinct communities (shown by nine individual colors). 
Table S6: Comparison of the calculated modularity index and variation of information between WT and protease variants using Louvain algorithm. The standard deviations from the networks of replica simulations are included.

\begin{tabular}{|l|c|l|}
\hline HIV-1 protease type & Modularity index (Q) & $\begin{array}{l}\text { Variation of Information } \\
\text { (WT-Mutant) }\end{array}$ \\
\hline WT & $0.594 \pm 0.002$ & 0.0 \\
\hline AM & $0.587 \pm 0.002$ & $0.2469 \pm 0.024$ \\
\hline ANAM & $0.603 \pm 0.001$ & $0.2985 \pm 0.009$ \\
\hline NAM & $0.601 \pm 0.003$ & $0.2415 \pm 0.046$ \\
\hline
\end{tabular}



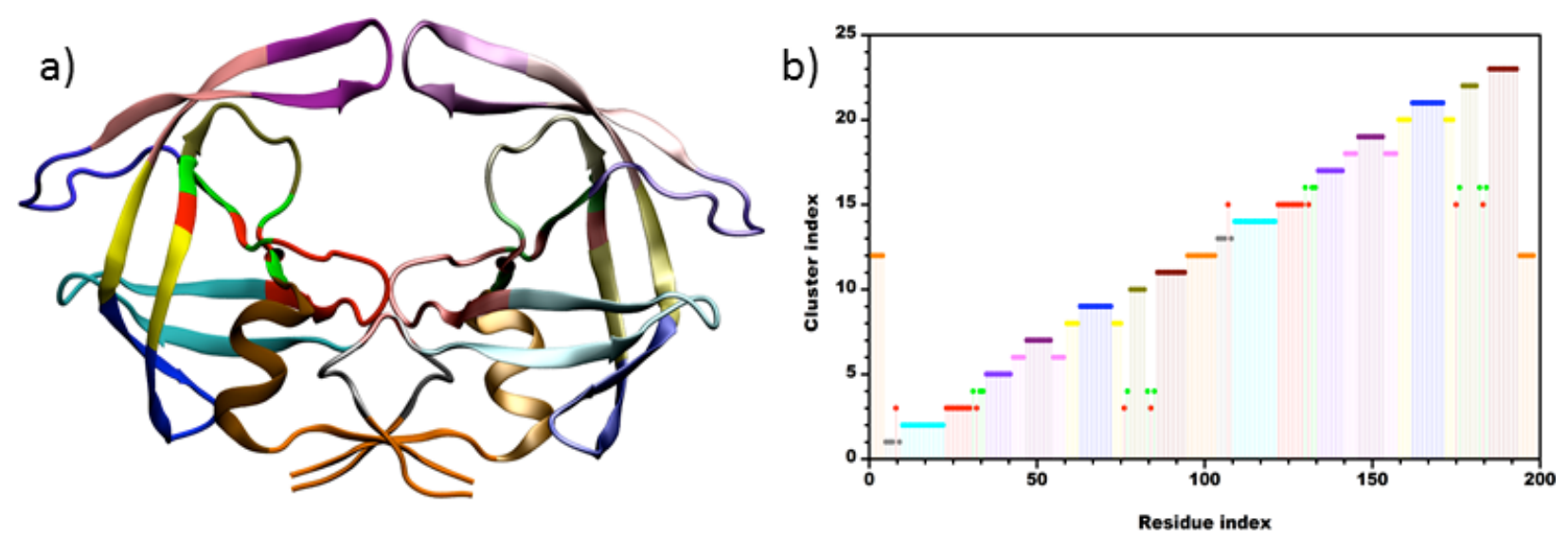

Figure S9: (a) Community structure of WT HIV-1 protease based on its structural and functional classifications. Each monomer is clustered into 11 different communities, plus a common community is considered that comprises all terminal residues. Thus, a total 23 different communities were present and are shown in 23 different colors. The names of these communities, based on their structural and functional roles, are tabulated in Table 1. (b) Cluster index (1 to 23) of each protease residue is shown. 

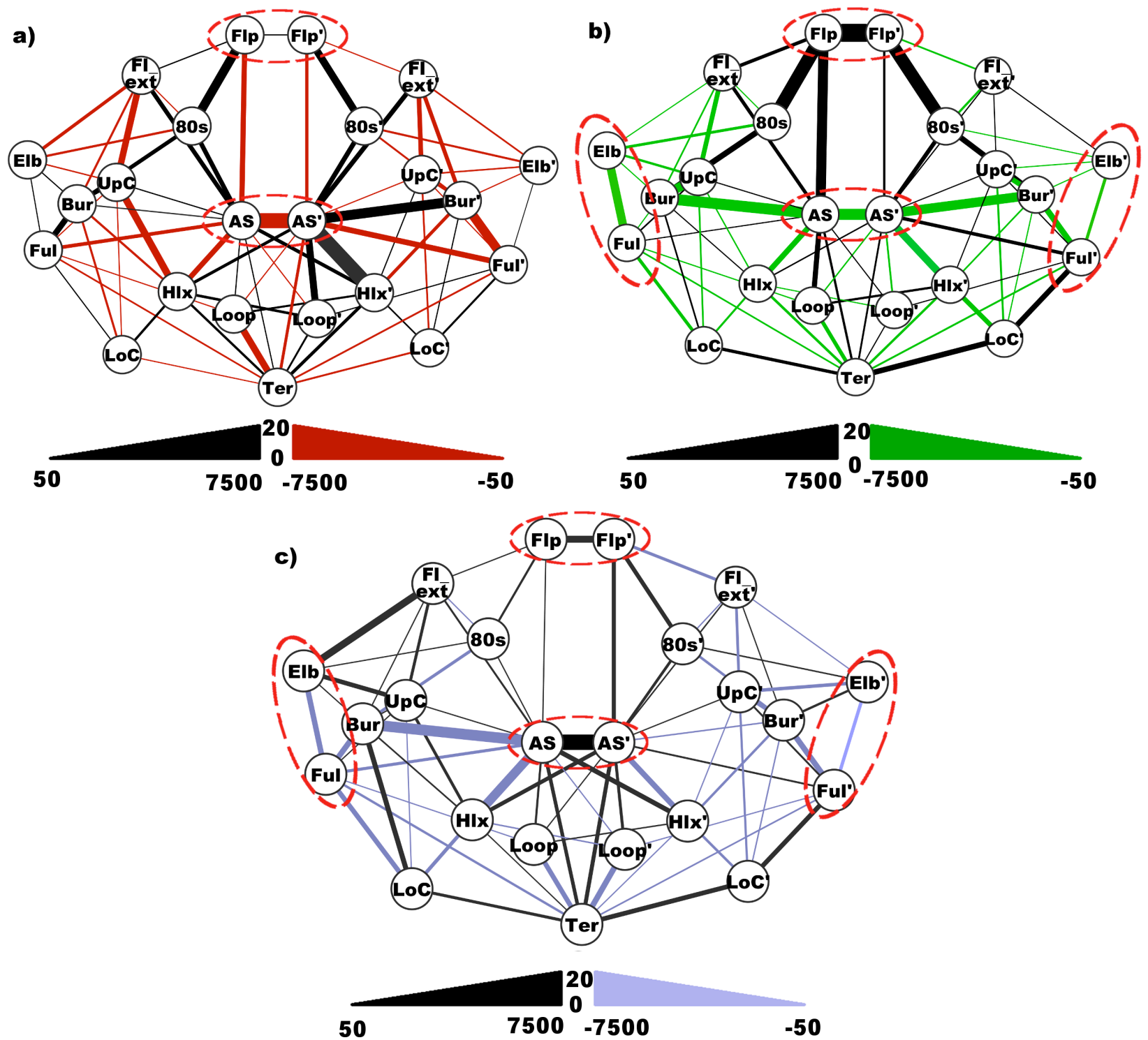

Figure S10: Change in information flow in the variant: (a) AM, (b) ANAM, (c) NAM_ALO relative to the WT protease (WT - variant). Color edges represent stronger, while black edges represent weaker inter-community communications in the variant than the WT. Width of the edges is proportional to the extent of strengthening/weakening of communications. Respective scale of edge widths is included and noteworthy inter-community edges are circled. 


\section{References:}

(1) Clune, J., Mouret, J., and Lipson, H. (2013) The evolutionary origins of modularity. Proc. R. Soc. B 280, 20122863.

(2) Fernandez, A. (2007) Molecular basis for evolving modularity in the yeast protein interaction network. PLoS Comput. Biol. 3, e226. 